\title{
CAD modelling of the human femur taking into account the structure of the bone
}

\author{
Andrei Horia Brănescu, ${ }^{1,}$, Ioana-Codruţa Lebădă², Vlăduț Mihuț ${ }^{1}$, Nenad Marjanovic ${ }^{3}$ and \\ Milan Rackov ${ }^{4}$ \\ ${ }^{1}$ Lucian Blaga University of Sibiu, Faculty of Engineering, No. 4, Emil Cioran Str., 550025, Sibiu, \\ România \\ ${ }^{2}$ Lucian Blaga University of Sibiu, Faculty of Medicine, No. 2A, Lucian Blaga Str., 550169, Sibiu, \\ România \\ ${ }^{3}$ University of Kragujevac, Faculty of Engineering, No. 6, Sestre Janjic Str., 34000, Kragujevac, \\ Serbia \\ ${ }^{4}$ University of Novi Sad, Faculty of Technical Science, No. 6, Dositeja Obradovića Str., 21102, Novi \\ Sad, Serbia
}

\begin{abstract}
The use of 3D models of the human bone system has great utility in the biomechanical study, due to the fact that these structures cannot be analyzed or tested in vivo. Within this paper, taking into consideration its real bone structure, the human femur CAD modelling is established. Firstly, a preliminary anatomical study is done, in order to highlight the fact that the thigh bone is a heterogeneous structure, consisting of several entities with different mechanical characteristics. The modelling starts from a homogenous 3D model of the femur, from which, every single separate entity is designed and finally they are assembled within a single product assembly. Therefore, the final result ensures a complex CAD structure, able to get assigned different material properties, specific for each entity this modelling furtherly allows the characteristic pathological structures studies of the referred entity or to simulate specific surgical interventions.
\end{abstract}

\section{Introduction}

The computer-assisted 3D human bones modelling is a very frequent method while elaborating biomechanical and bioengineering researches [1-7]. Developing the 3D models for the human lower limb assure good premises regarding the comprehension of the shape and kinematics of these complex geometric structures, biomechanical analyses of the human 3D foot assembly, for healthy subjects and for pathological conditions as well, alongside with virtual surgeries simulations [4-7]. These specific methods allow elaborating studies and further design of specialized devices, used within surgeries [1-3,8]. The present paper fits within this context, being focused on the human femur 3D modelling. Due to the fact that the nowadays goal regarding the anatomical CAD-CAE approaches [7] is to accomplish more

\footnotetext{
* Corresponding author: horia.branescu@ulbsibiu.ro
} 
accurate results, a very important role is played by the modelling correctitude of the anatomical structures, as in the bone, cartilage and tendons structures. The human skeleton is the main support framework of the body, within there are found several types of bones, categorized by their shapes: long, short, flat and irregular bones. This study aims on the long bones real structure, revolving around the most relevant bone within the category, the human femur, also called thighbone. The bone will be divided into several entities, each characterized by the suitable structure and mechanic characteristics.

\section{The real structure of the bone}

For a better comprehension of the real femur structure, it's lack of homogeneity, diverse mechanical characteristics and sub-structuration within it, a bibliographic study has been elaborated [9-13].

Based on the previously mentioned study, several facts have been highlighted regarding the constructive approach of the bone; the thighbone can be discretized into three main subelements: the two epiphysis and the diaphysis (or shaft), due to their different geometric and dimensional aspects. The diaphysis can be assimilated with a tubular structure, filled with the marrow, connecting the two epiphyses. The mechanical point of view is another main difference between the three elements, due to their different functional purpose.

Within the bone structure, there are four structural elements, starting from the mentioned tubular cavity called the medullar channel, the cortical and trabecular bone and finally, the cartilage. The disposing of the elements is presented in Fig. 1.

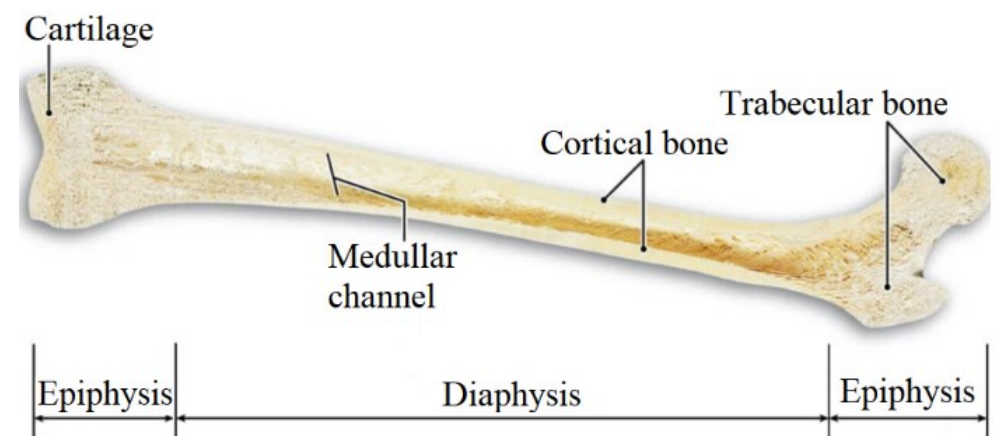

Fig. 1. Thighbone structural elements.

The studied bibliography regarding CAD modelling describes the shaft area as a rather homogenous structure, most of it being hard cortical bone. Several CAD studies consider the tubular area being designed in an elliptic shape, with a constant thickness, where others describe it as an offset from the external surface of the bone, with variable thickness, narrower near the epiphyseal zones [14], [15].

In order to elaborate an accurate 3D model of the bone, we must take into consideration the different mechanical properties of the long bones, particular for each mentioned area. Obviously, there is a great gap between values from individual to individual, depending on the biological aging, diseases etc. One of the best non-invasive methods for analyzing the particular mechanical properties of a subject's bone, is the radiography computer processing. [16]. This method is defining the mechanical characteristics of the bone by calculating the bone density base on the grey-tone [17].

Based on numerous research articles and studies, we concluded to a set of values which are of a healthy male subject, centralized in Table 1; data was harvested from [18-20]. This data will play a significant role in the further CAE modelling. 
Table 1. Mechanical characteristics of the bone's structures.

\begin{tabular}{|c|c|c|c|}
\hline & Cortical bone & Spongious bone & Articular cartilage \\
\hline Young Module (E) & $16 \mathrm{GPa}$ & $0.9 \mathrm{GPa}$ & $0.0125 \mathrm{GPa}$ \\
\hline Poisson coefficient & 0.325 & 0.18 & 0.225 \\
\hline $\begin{array}{c}\text { Transversal elasticity } \\
\text { module }(\mathrm{G})\end{array}$ & $3.4 \mathrm{GPa}$ & $0.25 \mathrm{GPa}$ & - \\
\hline Density & $1.825 \mathrm{~g} / \mathrm{cm}^{\wedge} 3$ & $0.725 \mathrm{~g} / \mathrm{cm}^{\wedge} 3$ & - \\
\hline
\end{tabular}

The main fact that must be highlighted from of our previously made brief study is the high complexity of the bone structure. In order to accomplish the goal to model this structure, we applied the constructive-anatomical entity method [18, 21-23]. This specific method assumes dividing the main structure into several separate entities, which are then assembled as convenient for the 3D designer. The fragmentation of the main structure must take into consideration plenty of aspects, regarding the medical actions that take place within each entity, the loading state of it etc. [24].

Based on the recalled method, the femur is divided into four entities: the two epiphyses (proximal and distal), the diaphysis and the articular cartilage; each entity has a different structure based on the anatomical particularities and mechanical solicitations, as following: the shaft will be considered a tubular shape of cortical bone, the epiphyses will be designed as a core of trabecular bone, covered with a cortical bone shell and finally the cartilage. After each entity is designed, the software allows to associate the suitable material characteristics for each one. [25].

\section{Real structure of the femur - 3D modelling}

The software that we used for our modelling is Catia V5R20 and has been done starting form a tridimensional femur model previously scanned. This specific model does not have a high degree of utility in the CAE approach, due to the fact that it is a homogenous structure and it can only be assigned a single type of mechanical characteristics, the initial model is presented in Fig. 2.

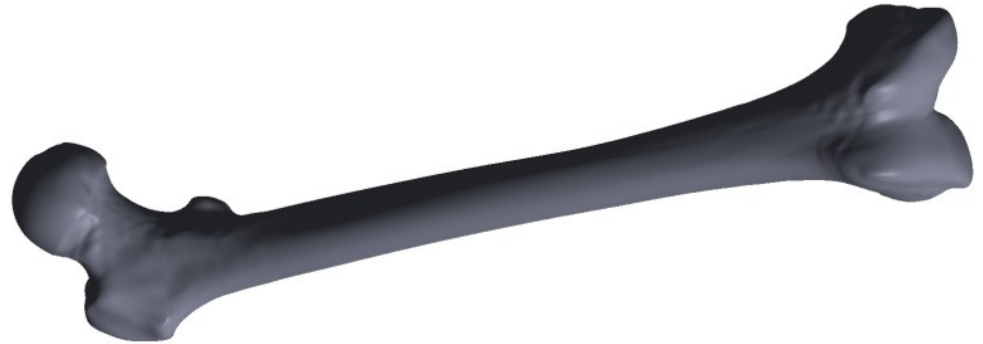

Fig. 2. Initial femur structure

In the following paragraphs, the whole method used to accomplish the real femur structure will be presented.

The first step was to establish the right sectioning path; this establishment has been done by pursuing the method presented in [26], as following, the greatest femur length for our scanned bone is $\sim 456 \mathrm{~mm}$, therefore by using the data within the mentioned paper, the femoral shaft will have a length between $289-422 \mathrm{~mm}$, we will further consider a medial value of $300 \mathrm{~mm}$ for the diaphysis; Fig. 3. 


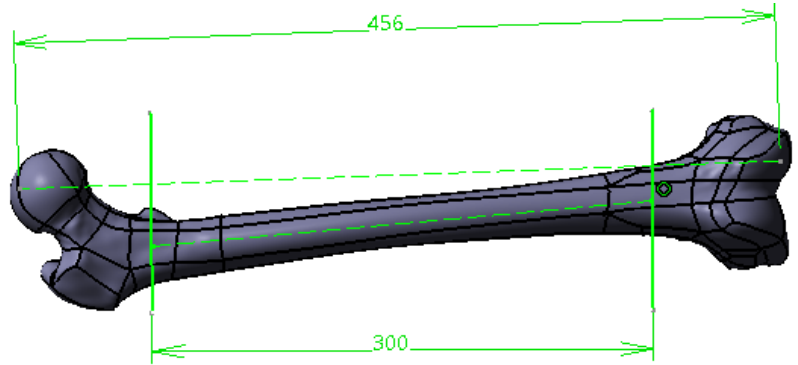

Fig. 3. Establishment of sectioning paths

After the sectioning paths are established, the bone will be cut and saved into three different parts: femur_d.catpart, for the diaphysis, femur_el.catpart, for the proximal epiphysis and femur_e $\overline{2}$.catpart for the distal epiphysis.

As presented, the anatomical-constructive entity of the proximal and distal epiphysis is structured in two layers, the spongy core and the hard shell. Starting from the femur_el.catpart, we will shift into the Generative Shape Design module in Catia, environment which allows us to "extract" all the exterior surfaces of the bone (all without the cut-out). After extracting all the surfaces, we will use the offset command, and shrink them with an offset of $2 \mathrm{~mm}$ (the hard cortical bone shell). The $2 \mathrm{~mm}$ value was considered by analyzing paper [27]. Fig. 4 and Fig. 5 presents the surface selection and offsetting.

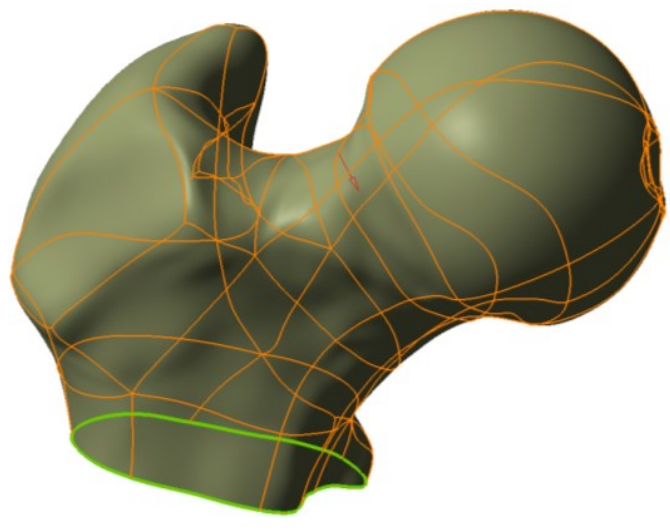

Fig. 4. Surface selection

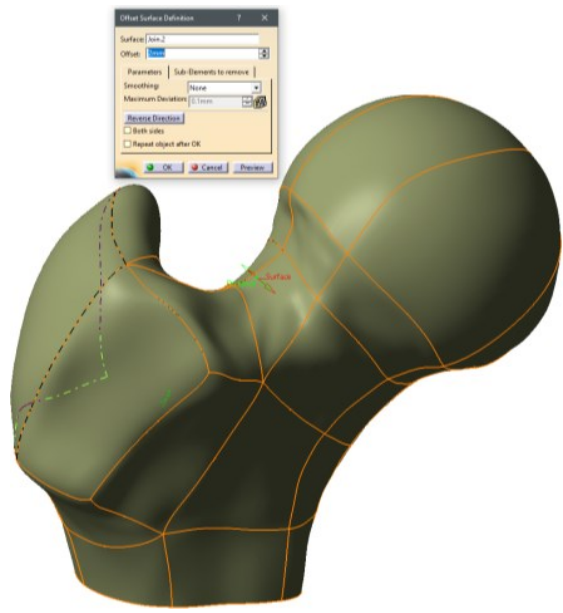

Fig. 5. Setting the $2 \mathrm{~mm}$ offset

Due to the high surface complexity, several manual adjustments have to be made, by using the commands "Heal" and "Fill", but once the whole shell is complete, the initial part will be cut with this offset surface, resulting the outer thin hard layer, as presented in Fig. 6. This part will be separately saved as femur_e1_2.catpart and by using the Boolean Operation "Remove", we will discard this part from the initial one, resulting the trabecular core of the epiphysis, as well saved individually as femur_el_1.catpart. The complete sub-element of the proximal epiphysis entity, containing the two layers, is presented in Fig. 7. The exact same methodology was used in elaborating the distal epiphysis, obtaining the sub-elements femur_e2_1.catpart and femur_e2_2.catpart respectively. 


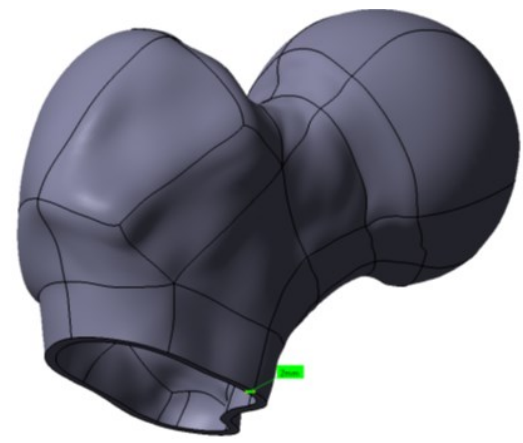

Fig. 6. Outer cortical shell

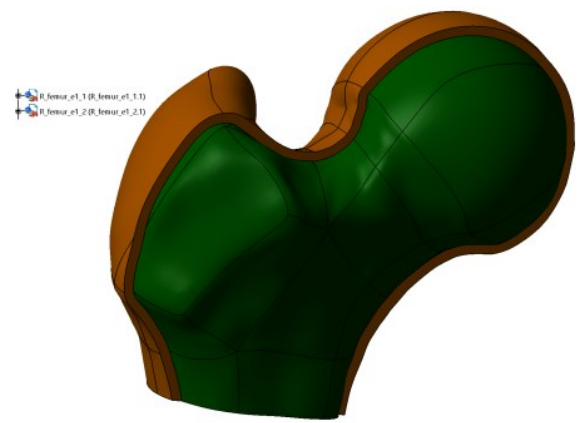

Fig. 7. Subassembly of cortical (orange) and trabecular (green) layers of the proximal epiphysis entity - section view

Regarding the shaft area of the femur, as previously presented, accordingly to numerous lately studies, the thickness of the cortical shell within the element varies, having a tendency of increasing the section bulkiness, as moving away from the two epiphyses, especially posterior and medial; this approach is more accurate than the basic elliptical medullar channel method. For our $300 \mathrm{~mm}$ diaphysis, we sectioned it with 14 planes, parallel to each other, every $20 \mathrm{~mm}$, Fig. 8 . Based on the studied bibliography [24, 28-31], we established the thicknesses of the shaft, starting from $2 \mathrm{~mm}$, near the two epiphyses, and ranging up to $6 \mathrm{~mm}$, in the middle of the bone. The process in developing the shaft was fastidious, requiring sectioning the bone with each plane, projecting the outer contour, isolating the curve and then remove the initial section, for each one of the 14 planes. Further, each 14 curves, adding the two on each primary cut, had to be offset with the specific value of the thickness. After each section is offset, by using the Removed Multi-sections Solid command within the Part Design module, we were able to discard the medullar channel. A sectioned view of the diaphysis is presented in Fig. 9.

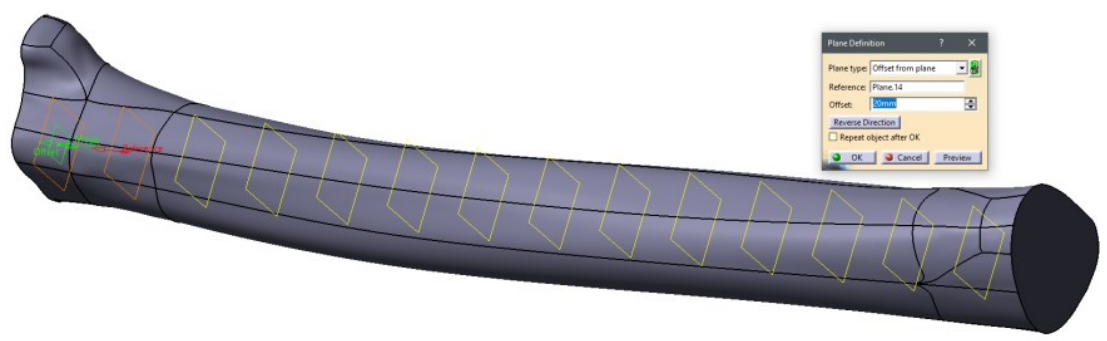

Fig.8. Defining the 14 sections for the further thickness variation of the shaft.

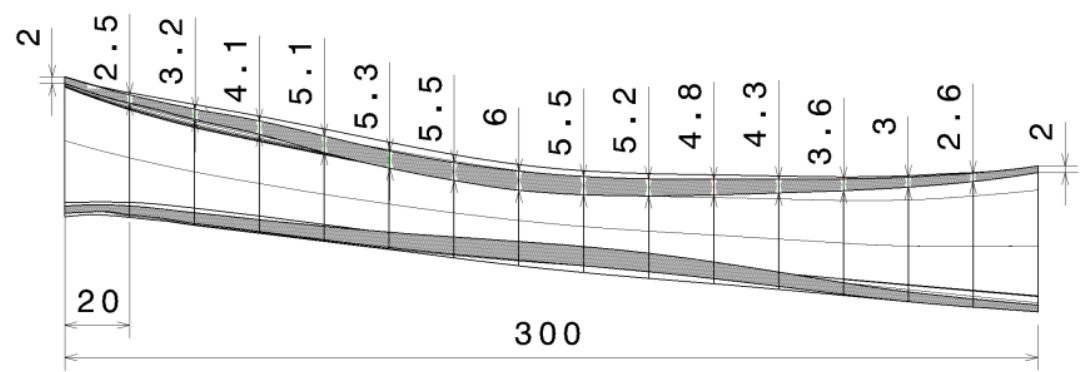

Fig. 9. Thickness variation of the shaft - longitudinal section view 
The usage between the outer elements in the human inferior leg takes place within the cartilage, therefore, the development of it presents high interest. The methodology of the 3D design regarding the cartilage element involves the same steps as the elaboration of the two epiphyses, by offsetting the contact surfaces with $2 \mathrm{~mm}$. Once the offset is done, we considered a $1 \mathrm{~mm}$ corner radius on the top contour, in order to remove the potential tension concentrators in the further Finite Element Analysis. The part was separately saved as cartilge.catpart, and is presented in Fig. 10.

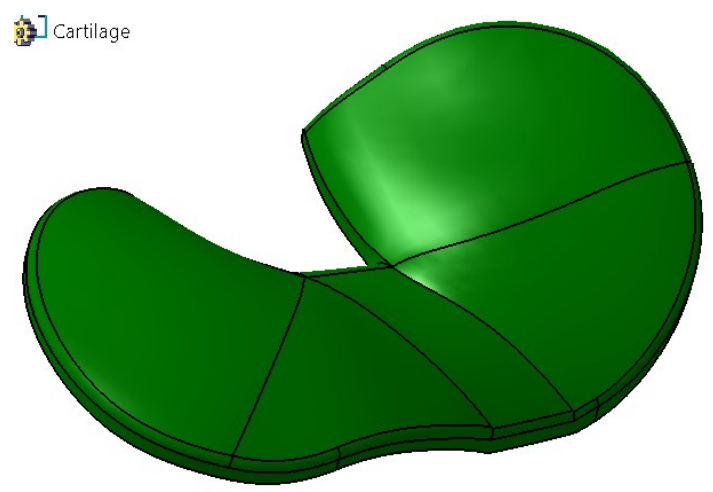

Fig. 10. Cartilage entity

The final step of the modelling of the real femur structure consists in the assembly of the six specific elements: femur_d.catpart, femur_e1_1.catpart, femur_e1_2.catpart, femur_e2_1.catpart, femur_e2_2.catpart and cartilage.catpart, into a new product, named F_1.catproduct, by using the Coincidence command within the Assembly design module, due to the fact that the elements share common elements as points and curves, resulted through the cutting and splitting process. Once the constraints are defined and the elements are fixed, the final structure is ready to be used; the final result is illustrated in Fig. 11 in an isometrical view, and in Fig. 12 in a sectioned view, where all the elements and offsets are displayed.

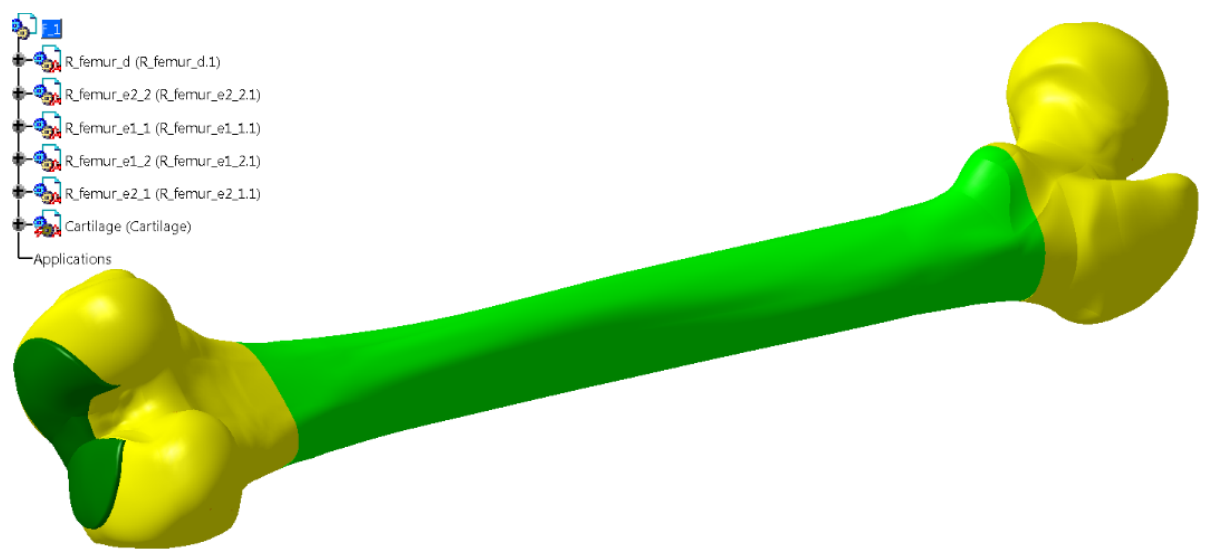

Fig. 11. Isometrical view of real femur

By assembling all the separate entities, we obtained a firm structure of the femur, on which can be assigned different material characteristics and materials, fact that will assure more accurate results of the simulations or analysis, compared to the initial homogenous solid bone. 


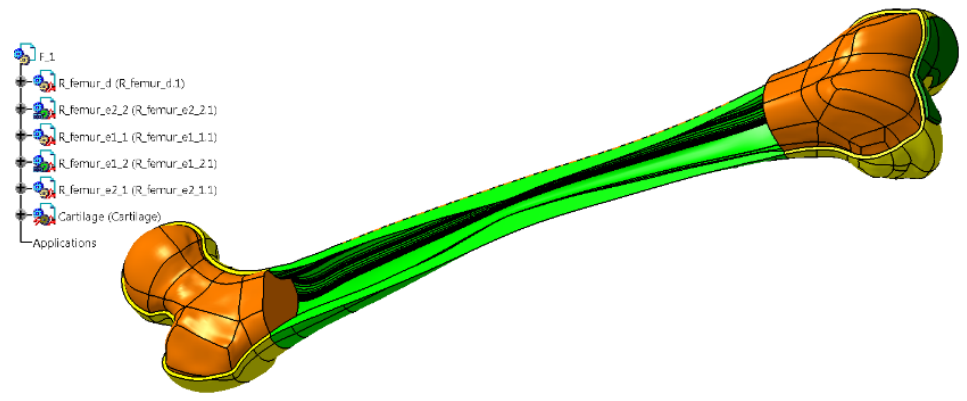

Fig. 12. Sectioned view of femur

The developed 3D model contains all the real anatomical entities, which can be further exploited in biomechanical studies as particular elements or as the whole assembly, for rather healthy or pathological conditions. Such a medical pathological condition in which the elaborated model can be used is to design the knee osteoarthritis, for further CAE studies or Finite Element Analyses. The knee osteoarthritis is a frequent medical condition among elder women, consisting in degeneration of breaking down of the cartilage.

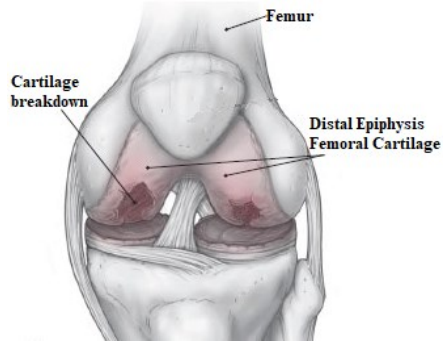

Fig. 13. Knee osteoarthritis.

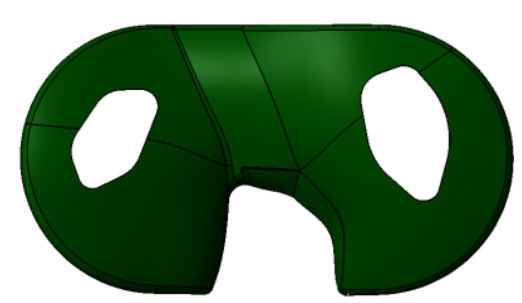

Fig. 14. Torn distal epiphysis femoral cartilage

The knee osteoarthritis condition, where the distal epiphysis femoral cartilage is torn from the joint, is illustrated within Fig. 13. By using data required from papers [32-33], we elaborated the approximate shape of the torn cartilage, and then, in the cartilage.catpart entity we reproduced it, result presented in Fig. 14. By assembling the cartilage entity with the other entities within the femoral structure, we obtain the final model of the medical condition, elaborated in Fig. 15.

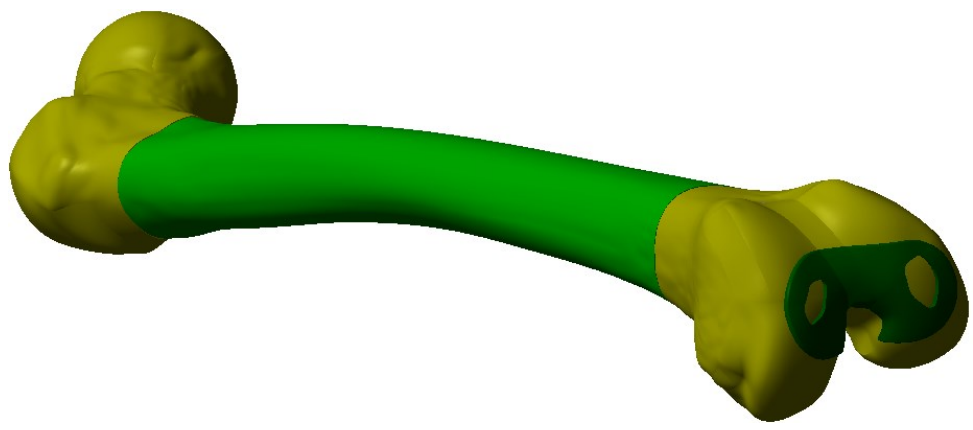

Fig. 15. Final assembly of the pathological condition

Due to the fact that this condition often requires joint replacement surgery, the real 3D femur model has high usefulness, for accurate development of the necessary plastic or metal prosthetics. 


\section{Conclusions}

The current paper provides important contributions in terms of the real CAD modelling of the femur. The main concept in developing the real bone structure was the MedicalAnatomical-Constructive entity, a relatively new and innovative concept, which assure firm and relevant results. This concept has its main entries the functional constructive entities (in this specific case: cartilage, epiphysis and diaphysis), which, alongside with their main specifications: shape, dimensions, mechanical solicitations, structure type and mechanical characteristics, is developed the proper modeling of the desired element, while taking in account the medical aspects as well (physiological particularities, affections or specific treatment strategies). Taking into consideration the high complexity of the structure, elaborating a 3D model is very useful in the further Finite Element Analysis, for a better comprehension of the real bone behavior, during the specific loading states within the gait and standing phase.

\section{References}

1. I. I. Cofaru, P. D. Brindasu, N. F. Cofaru, Applied Mechanics and Materials, ISSN: 1660-9336, ISSN/ISO: Applied Mechanics and Materials, (2013);

2. N. F. Cofaru, I. I. Cofaru, R. E. Petruse, M. Rackov, M. Blagojevic, N. Marjanovic, MATEC Web of Conferences 299, 03011 (2019);

3. N. F. Cofaru, I. I. Cofaru, V. S. Oleksik, A. Pascu, M. V. Dragoi, KOD2018 IOP Conference Series: Materials Science and Engineering, 393 (2018);

4. N. F. Cofaru, M. D. Roman, I. I. Cofaru, V. S. Oleksik, S. R. Fleaca, Applied Biosciences and Bioengineering, Applied Sciences (ISSN 2076-3417), Special Issue "Biomechanical and Biomedical Factors of Knee Osteoarthritis", (2020);

5. N. F. Cofaru, I. I. Cofaru, V. Marjanović, N. Marjanović, M. Blagojević and R. E. Petruse, CAD modeling of the closing High tibial osteotomy, (2019);

6. I. I. Cofaru, N. Cofaru, Computer assisted approach to the axial deviations of the human lower limb, Academic Journal of Manufacturing Engineering - AJME, (2017);

7. I. Huzu, N. F. Cofaru, 657, Engineering Solutions and Technologies in Manufacturing, (2014);

8. I. I. Cofaru, The CAD modelling of the human tibia affected by form deviations, Fascicle of Management and Technological Engineering, Issue 1, (2014);

9. Z. Yosibash, Comput. Methods Appl. Mech. Engrg, pp. 169-184, (2012);

10. K. Chan, Handbook of Footwear Design and Manufacture, pp. 3-26, (2013);

11. K. Wesker, Thieme Atlas of Anatomy: General Anatomy and Musculoskeletal System. (2007);

12. S. Standring, Gray's Anatomy, thirty-ninth ed. Elsevier Churchill Livingstone, Edinburgh. J. Orthop. Res. 13 (4), pp. 609-614, (2005);

13. Z. Milojević, S. Navalušić, R. Obradović, M. Milankov, M. V. Drăgoi, L. Beju, Academic Journal of Manufacturing Engineering 8 (1), pp. 73-78, (2010);

14. L. Voo, Johns Hopkins Apl Technical Digest, 25, Number 3, (2004);

15. M. Peacock, D. L. Koller, D. Lai, S. Hui, T. Foroud, M. J. Econs, Bone, 37, pp. 467-473 (2005);

16. S. Ćuković, R. E. Petruse, G. Meixner and L. Buchweitz, 2020 IEEE International Conference on Bioinformatics and Biomedicine (BIBM), pp. 1926-1931, (2020);

17. E. Schileo, J. Pitocchi, C. Falcinelli, F. Taddei, Bone, 136, (2020);

18. I. I. Cofaru, P. D. Brîndașu, N. F. Cofaru, L. Roman, Computer Simulation Paradigm Regarding the Structure and Mechanical Characteristics of Human Long Bones (2013);

19. Y. Wang, D. W. C. Wong, M. Zhang, Ann. Biomed. Eng. 44 (1), pp. 213-221, (2016); 
20. A. Perrier, Biomechanics of Living Organs, Chapter 25, pp. 545-563, (2017);

21. I. I. Cofaru, MATEC Web Conf, 121, No. 4004, (2017);

22. I. I. Cofaru, E. I. Huzu, Generalized Modelling of the human lower limb assembly, The 1st International Conference for Doctoral Students, (2013);

23. I. I. Cofaru, Biomechanic of the opening tibial osteotomy, Fascicle of Management and Technological Engineering, Issue 1, (2014);

24. H. Arjmand, M. Nazemi, S. A. Kontulainen, C. E. McLennan, D. J. Hunter, D. R. Wilson, J. D. Johnston, Scientific Reports, 8, 11478, (2018);

25. M. Polguj, K. Bliźniewska, K. Jędrzejewski, A. Majos, M. Topol, Morphological study of linea aspera variations: Proposal of classification and sexual dimorphism, (2013);

26. J. O’Connor, L. M. Alves Borges, F. P. Duda, A. G. Barbosa da Cruz, Growth and reabsorption in biological tissues, (2015);

27. K. Engelke, S. Prevrhal, H. K. Genant, Macro- and Microimaging of Bone Architecture, (2008);

28. K. C. N. Kumar, S. Chavadaki, V. Chaudhry, D. P. Singh, A. Shaikh, Material Proceedings, 26, Part 2, pp. 356-363, (2019);

29. J. H. Gosman, Z. R. Hubbell, C. N. Shaw, T. M. Ryan, The anatomical record, 296, pp. 774-787 (2013);

30. I. Goda, M. Assidi, J-F Ganghoffer, Cosserat Anisotropic Models of Trabecular Bone from the Homogenization of the Trabecular Structure: 2D and 3D Frameworks, (2013);

31. N. Chantarapanich, S. Rojanasthien, B. Chernchujit, Journal of Orthopaedic Science, 22, pp. 703-709 (2017);

32. D. M. Blaustein, E. M. Philips, Essentials of Physical Medicine and Rehabilitation (Fourth Edition), pp. 391-398, (2019);

33. S. D. Waldman, Pain Medicine: A Case-Based Learning Series, (2019). 